\title{
A Methodology for Optimised Cultural User Personas Experience - CURE Architecture
}

\author{
Markos Konstantakis, Konstantinos Michalakis, John Aliprantis, Eirini Kalatha, Efthymia Moraitou, George Caridakis \\ Department of Cultural Technology and Communication, \\ University of the Aegean \\ Mytilene, Greece \\ \{mkonstadakis, kmichalak, jalip, ekalatha, gcari, e.moraitou\}@aegean
}

\begin{abstract}
Cultural Heritage Institutions (CHI) are increasingly aiming at enhancing their visitors' experiences in a personalised, immersive and engaging way. A personalised system for cultural heritage promotion potentially adapts, in terms of relevance, content and presentation according to the user's interests and needs. However, since a typical visit may be short and unrepeatable, the identification of user's profile must be quick and efficient, ensuring the successful respective personalisation process. Current paper discusses a methodology for cultural user personas extraction and identification. The CURE approach eliminates the requirement of explicit user input via registration or similar data acquisition methods and involves three main stages: data acquisition from the user's online and social activity, reasoning regarding persona similarity and finally data and experiences reuse from previous visits. Regarding the constructed personas, the proposed approach continuously adapts and refines the personas features from data gathered during multiple cultural experiences and accordingly creates, deletes or merges personas in case of significant deviation, poor correlation and convergence respectively.
\end{abstract}

User Personas, Personalisation, Cultural mobile applications, Evaluation, Cultural User Experience

\section{INTRODUCTION}

Peter having read some favourable posts on social media, decides to visit a new exhibition in one of the museums of the city. Standing on the entry of the museum, he carries expectations of an interesting cultural visit, yet he is unsure on how to start. Peter asks museum employees whose first advice is to connect through his mobile device to the museum's Facebook page in order to download the application, which offers a personalized path. As Peter follows the suggested route into the physical museum space, a path through his mind is walked as well, discovering personalised experiences.

Recently, interactive experiences in Cultural Heritage Institutions $(\mathrm{CHI})$ are produced in an increased pace. One of the main purpose of this interest has been to enhance user's interaction with cultural objects by adjusting the cultural experience to his expectations and needs, such as autonomy, competency, stimulation, relatedness and popularity (Hassenzahl, 2008; Vermeeren et al., 2010). The importance of high quality User experience (UX) is increasing as user's base of current trends in computing is constantly growing and shifting. Thus, the evaluation of the visitors' experiences using new technologies (Konstantakis et al., 2017) (such as Ubiquitous computing, Internet of Things, Context Awareness) is of importance for $\mathrm{CHI}$, but as yet unexplored (Chianese \& Piccialli, 2015).

Since digitisation is changing the cultural landscape, $\mathrm{CHI}$ have been struggling with their exhibits and their environments. Previously, the cultural space has acted as a site of preservation, storage and exhibition, as well as a center of information dissemination. Currently, they have changed to take on more concrete functions such as enabling and facilitating active learning and visitors' engagement with exhibits, as well as active collaboration on information seeking and sharing between visitors (Othman, 2012). The convergence of the web has made the exploration of cultural heritage $(\mathrm{CH})$ a continuous process, starting before the visit and ideally never ending, as the user is able to plan the visit online, visit the site, and then "revisit" places of interest online again.

In this context, applying a methodology which will engage the user into the provision of profiling information might in fact decide the success or failure of the cultural experience. The user profile initialisation is also an important aspect for personalised cultural applications and a particularly challenging task to tackle. Thus, in the field of $(\mathrm{CH})$ 
there have also been efforts to minimise the problems of personalisation initialisation by using 'personas' (Kenteris, Gavalas \& Economou, 2011).

In this paper, we propose the CURE methodology (Cultural User peRsonas Experience) for enhancing user personas experiences in $\mathrm{CHI}$, by incorporating data acquisition techniques from social activity or previous experiences, rather than explicit user input. In the remainder of the paper we present the related work and studies and we analyze the CURE methodology for the identification of user personas. Finally, we describe the backend architecture of the CURE proposed methodology and afterwards we discuss our future plans and directions.

\section{RELATED WORK}

The technologies used in some studies (Cheverst et al., 2000; Vassilakis et al., 2016) were tailored to suit the needs of particular cultural spaces providing a wide range of technological means to citizens and venue visitors in a highly personalised manner. Nevertheless, users found that is harder to use a system with too many options available.

Some researchers have analysed the visitor's personalised experience from a philosophical perspective (Ardissono, Kuflik \& Petrelli, 2012; Findlater \& McGrenere, 2004) addressing the challenges of socialisation and collaboration in small and large-size communities, whilst others have addressed different aspects of the visitor experience empirically, including the cognitive, intellectual and emotional aspects (Antoniou \& Lepouras, 2010).

In addition, others have investigated the interaction between individual visitors and exhibits, or between visitors in groups and exhibits, collecting information prior to the visit (Falk, 2006; Kuflik et al., 2011).

Furthermore, in the field of $(\mathrm{CH})$ there have also been efforts to minimise the problems of personalisation initialisation with the use of 'personas' (Bonis et al., 2009; Roussou et al., 2013) and to improve the usability of mobile applications through context awareness, resulting in a better UX (Davies, 2007). Still there are issues such as the exact number and defined variables of the selected personas. Moreover, in some cases the user does not correspond to any of the personas or match equally with more than one, a significant problem which need to be addressed.

\section{PERSONALISATION}

Personalisation (Antoniou \& Lepouras, 2010) is based on the assumption that an application can understand the user's needs, while its success relies greatly on the accurate elicitation of the user profile. In a typical cultural space visit the users' time is limited. It may last as little as a few minutes, and the users might only visit the cultural site once.

An increasing number of $\mathrm{CHI}$ around the world use personalised, mostly mobile, guides to enhance visitors' experiences, attract new visitors and address the needs of a diverse audience. The use of personalisation technologies has now become very common in $\mathrm{CHI}$. However, there is still a lack of understanding about how visitors interact simultaneously both with such methods and the exhibits.

A unique aspect of $\mathrm{CHI}$ visitors is the diversity that they present, an attribute which becomes a major challenge for these venues to meet their visitors' needs. Yet, the question still remains "How can they address the variety of interests and needs of all their visitors?".

According to (Falk, 2006; Morris, Hargreaves \& Mclntyre, 2004) there are four different modes of behaviour among visitors in $\mathrm{CHI}$, especially when they select and engage with the exhibits: 'browsers', 'followers', 'searchers' and 'researchers'. These four types of visitor may prefer different kinds of information presentation and therefore different technologies can be implemented/used.

Browsers, for example, do not require as much information as researchers because they only browse and select exhibits that most appeal to them. They are motivated by their own curiosity, thus they wander around the exhibition until they find an object that catches their attention. According to Falk (Falk 2006), they can be described as "science lovers", "learners", "discoverers" or "curious people". Therefore, even though it is crucial for the personalisation techniques to identify browser's interests, their path in the exhibition might be unpredictable.

On the other hand, Researchers require more explanation about each artefact in the exhibition and may require extra information related to the exhibits. They follow their own path in the exhibition and usually pick specific objects based on their indepth knowledge of the subject, seeking additional and expert information. Although they may already possess a strong knowledge and interest in the exhibition's objects, they are equally motivated in learning not only additional information but also how this information was extracted and validated. As a consequence, researchers' background and knowledge is key to a successful personalized experience.

Followers, in contrast, only follow what has been provided to them and usually will be happy with the use of the mobile guide provided by the $\mathrm{CHI}$. Like Browsers, they can be characterized as passive consumers, and they are satisfied by letting the museum to select and describe the objects which 
illustrate better the theme of the exhibition. Furthermore, followers lack contextual knowledge of the exhibition and require easy access to information related to the object, while also they tend to move along with groups or family. So, it is unlikely that followers' experience will be further enriched by a personalized application.

Finally, a Searcher is quite different from the other groups because he prefers to search the exhibit based on keyword(s) rather than the thematic presentation. They may also rely on the museum to provide them with the basic objects of the exhibitions but they want to satisfy their personal interests. Like the researchers, searchers have also a level of pre-existing knowledge and they are interested in the whole exhibition and they want to consume all the available information available in the museum. Searchers and Researchers are described as reactive visitors and they can be strongly benefited by a personalized experience.

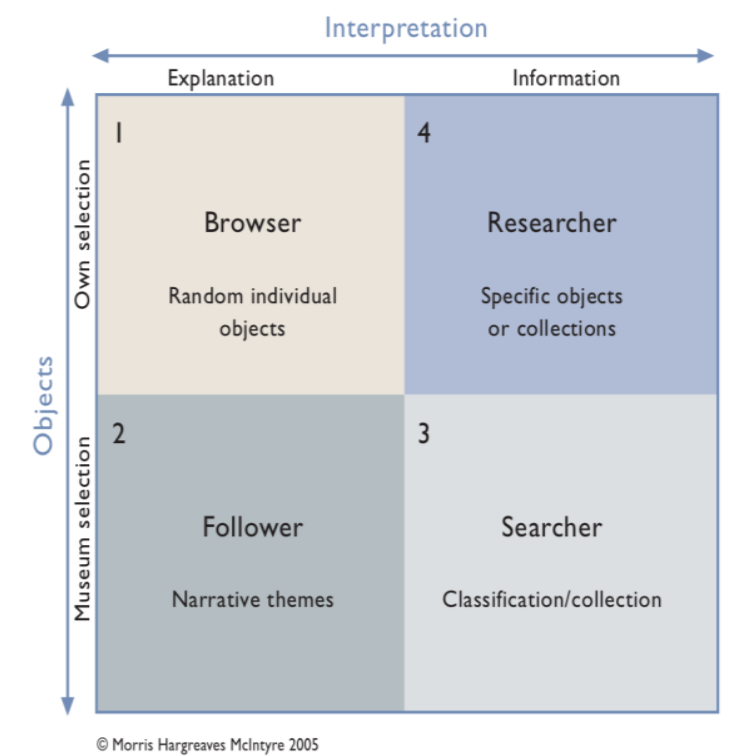

Figure 1: The 4 different types of visitors

Additionally, the authors in another study (Walsh, Clough \& Foster, 2016) identify the ways in which users of Digital Cultural Heritage (DCH) systems and services have been categorised. They proposed that it may be better to categorise users by expertise, than by label or user type. Alternatively, it could be applied some combination of both, since it would potentially enable the delivery and creation of more value-added services.

\section{CURE METHODOLOGY}

\subsection{The cold start problem - User Personas approach}

Although personalisation is useful in $(\mathrm{CH})$, creating correct visitor profiles is a rather demanding and sometimes intruding task basically due to the short duration of the visits and the fact that most visitors might only visit a specific institution only once (Vassilakis et al., 2016).

Within these time restrictions, visitor profiles need to be created quickly and effectively in terms of their appropriateness for the different visitors. The problem is that although visitors enjoy the benefits of personalisation in $(\mathrm{CH})$, they are at the same time reluctant at dealing with form-filling activities. Therefore, researchers have to become more creative in applying indirect approaches for the collection of the needed information for the creation of user profiles.

The design of personas as 'fictional' characters is considered as a very consistent and representative way to define actual users and their goals. However, it is important to clarify the exact (minimum) number of personas in each occasion in order to focus on the visitor profiles to be examined.

Moreover, in many cases these profiles are designed and built based on user input (surveys and questionnaires, records of user's patterns and moves), and as a result they represent only the average visitor profiles. Additionally, input data is not always in correspondence with the actual desires and interests of the user. What if the visitor does not really know in what he is most interested or visits a museum for the first time?

To be more accurate on the design of user personas, there is the need to rely on different sources for users' information, without distracting them from their cultural experience. Hence, during the start of a system, i.e. when a user is new to that system, the system fails to recommend paths and directions initially. This problem is called the cold start problem in personalisation.

Cold start problem is a well-known problem in recommender systems. Many solutions and methods are proposed to address this issue. Common recommendation strategies are based on association rules and clustering techniques (Sobhanam, 2013), social information (Zhang et al., 2010; Noor \& Martinez, 2009), ontological knowledge classification (Noor \& Martinez, 2009) and hybrid user modelling approach (Wang et al., 2008).

The proposed methodology will adopt Noor's and Martinez's ontological knowledge of social information and takes into consideration alternative sources of input from the user's digital and social activity. Applying reasoning techniques regarding persona matching, CURE performs an accurate identification procedure without any previous data concerning the user, thus partially addressing the cold start problem. 


\subsection{Identification of User Persona: Methodology steps}

Personalised applications need to be able to identify user's personas, i.e. their set of characteristics that defines their needs and expectations from the application.

Although an explicit request to the user, in order to select an appropriate persona, would seem to work, there are some explicit disadvantages such as the inconsistency of users' choices and their annoyance. Ideally, the application should be able to identify the User Persona without any user input or intervention and customise its functionality accordingly. Our work aims towards that direction, by proposing a methodology of identifying User Personas in a cultural context.

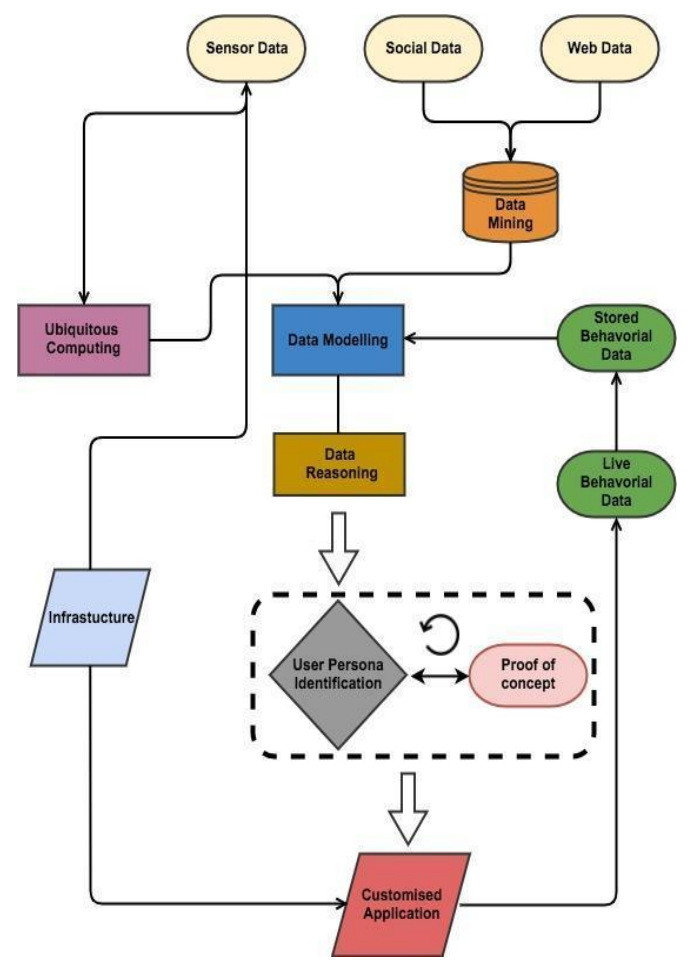

Figure 2: The CURE Methodology

The CURE methodology involves certain stages that are highly influenced by the data cycle of Context Aware Computing (Perera et al., 2014), stressing the correlation between this procedure and other context aware functionalities that are applied in similar situations.

These stages are: data acquisition, reasoning and dissemination. The data flow is shown in Figure 2.

Data acquisition: The first stage corresponds to data acquisition from multiple sources and involves the following two modules.

\section{- Data mining}

Data mining acquires profiled data which can be found from various repositories (mobile device, social media, other open access web data). Data mining techniques and natural language recognition are required to retrieve the profiled data of a user. Privacy and security of personal data will need to be addressed as well in this procedure. Typical issues with personalised applications are mining of personal data and ethics accompanying such procedures. Although trust from users is hard won, it should be sought by informing the users about the exact type of data collected and their exact use, as well as ensuring that no sensitive personal data will be stored.

\section{- Ubiquitous Computing}

The ubiquitous computing paradigm integrates sensory networks and context aware procedures to apply a personalised functionality in applications and devices. Specifically, in CURE there are two sources of behavioural data: (a) data retrieved from sensors of the mobile device (during the current or previous cultural visits) that allow a refinement of the user persona associated with the user and (b) data extracted from the sensory infrastructure of the cultural space, concerning user's activities and behaviour establishing a more effective connection between user and the cultural space. Due to lack of sensed data (e.g. first visit) or the uncertainty of interpreting behaviour, this input stream may have the least or the most weight on the identification algorithms. Furthermore, the ubiquitous characteristic of transparency is beneficial in this context, since we do not wish to burden the cognitive load of the visitors.

Data modelling / reasoning: The second stage includes the processing of the multiple data sources whose wide complexity and diversity will need to be modelled and reasoned. Sentiment analysis will be performed on the profiled and behavioural data, extracting usable information about the user profile.

Eventually, the user with a customised user persona, allowing a better understanding of his needs and goals. The original 4 user personas according to Morris et al., (2004) will be used as a basis which will be enhanced according to specific characteristics of each cultural user persona.

Data dissemination: The specialised persona of the user will be disseminated to the cultural application, allowing a customizable presentation of the various activities and attractions of the cultural place. Furthermore, as user explores the cultural application, the latter will continue to observe and collect behavioural data, sending feedback to the data modelling and reasoning components, allowing for further refinement of user personas and more efficient customisation of the cultural experience.

This cycle of persona identification will be continuously performed during the whole cultural visit of the user, eventually storing the identified persona for future use. Furthermore, the 
infrastructure of the cultural space also acquires the identified user persona and accordingly customises the user experience. Consequently, the whole cycle runs in a ubiquitous ecosystem where user, digital tools for artefacts and other infrastructure adapt to each other.

The architecture will also integrate a method for evaluation of the persona identification procedure. A specialised component is responsible for the collection of manual data concerning the user that will be checked against the output of the identification methodology. The differences between correct (manual) and identified personas will allow for an effective tuning of the algorithm so that it performs in a high level of accuracy.

\section{THE CURE ARCHITECTURE}

The cultural heritage experience is being viewed as an ongoing lifelong experience: curators and cultural researchers are continuously looking at how visitors can be captured and retained over time, both online and onsite.

Users' personalisation can play a major role by reasoning on past experience and other daily and contextual characteristics, making the current cultural heritage experience a link in a lifelong chain.

This creates a series of challenges that accompany lifelong experience user modelling in general: collecting data, remembering and forgetting (as the user's characteristics change), privacy and user control. This lifelong experience through the user personas identification is the basis of our CURE methodology. The CURE components will be built to form the back-end environment (Figure 3).

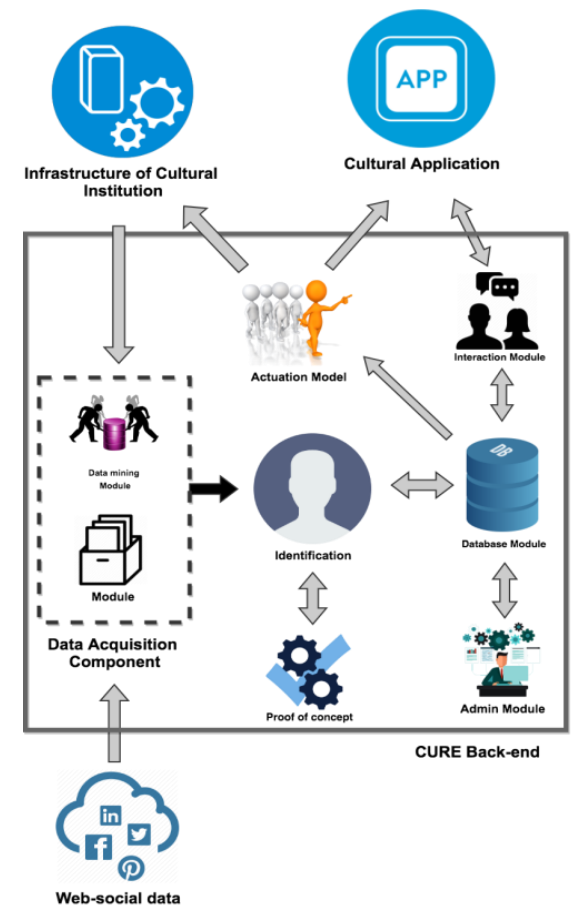

\subsection{The CURE back-end}

The CURE back-end hosts the information repository, as well as all services needed to support the visitor's application runtime. Also, it encompasses a repository for the storage, organisation and retrieval of cultural information and metadata, while it additionally hosts the CURE modules for delivering the needed functionalities to the application in the $\mathrm{CHI}$.

To accommodate the stages listed in the previous architecture, the CURE back-end functional modules comprising the system are the following 6 modules:

Data acquisition component: It handles data acquisition and modelling, sending ontologies and modelled data to the user persona identification module. The data are either mined from the web and social network concerning the user's cultural preference or collected from the physical infrastructure of the $\mathrm{CHI}$ (sensors measuring environmental or user variables).

Administration Module: This module can be used exclusively by the system administrator performing important operations for system management.

DB (Database) Module: This module is responsible to store and manage all the produced information that is relevant to the users. It communicates with all the modules while having no connectivity with outside elements for security and sustainability reasons.

User Persona Identification module: In this module, the main algorithms of data reasoning are executed, inferring the parameters of the persona of the current user. The input is retrieved from the Data Acquisition component and the output is stored to the Database module for further use. The module constantly updates the persona as more behavioural data arrive.

Interaction module: This module is responsible to retrieve interaction data. During his visit, the user will interact with elements of the cultural application, whose actions will feed the back-end with more data on his preferences and will allow further customisation of the UX.

Data dissemination module: This module handles all the necessary communication with the cultural application and the infrastructure of the institution about the customisation actions needed to enhance the user experience of a specific user or group of users. According to the information stored in the DB module, certain actuation calls are sent to the actors involved in providing the related cultural content and thus the personalised cultural user experience (e.g. the screen of an artefact near the user).

Figure 3: The CURE Architecture 


\section{USE CASE}

Let us return to Peter who is visiting another museum that integrates the CURE architecture. Peter has been identified by CURE as a "Follower", based on his previous visits to cultural spaces. His profile incorporates the basic characteristics of the persona (i.e. following suggested paths) while also including personal traits such as his preference for modern art exhibits and paintings. Also, his average visit time is higher than the general average time of the visitors.

Upon entry at the museum, Peter is automatically registered to CURE platform. The museum's Application (App) requests Peter's profile, initiating the personalisation procedure. Furthermore, CURE requests access to his latest social media posts, to apply sentiment analysis and possibly extract opinions that can be used in this context. Indeed, his twitter comment "Today is cultural day! I really want to check Beja's new artefact" indicates which exhibit will be at the center of Peter's attention. This information is also sent to the App.

Taking into consideration the profiled data disseminated from CURE, the App designs a personalised route for Peter. Since he is a follower, Peter is likely to accept the App's proposed route and start his visit by following the path, which is adapted to (a) his average visit time, (b) his cultural preferences and (c) any opinions mined from social posts. The App is not responsible for gathering personalised data but instead to customise predefined routes and procedures according to the persona characteristics identified by CURE.

As a "Follower", Peter is expected to follow the path suggested by the App. Yet, during this visit, Peter shows independency above a certain threshold, which adds some "searcher" traits to his persona. More specifically, he tends to ignore certain types of exhibits, while skipping sub paths and diverting his route to other locations. This information is extracted from Peter's mobile location sensors and from the museum's sensory infrastructure.

CURE (executed at the background) extracts the behavioural data and repeats the personalisation procedure, enhancing the original persona with Peter's specific preferences of the current cultural space and its exhibits. The modified and updated persona is disseminated to the App, which redesigns the paths and the functions provided to Peter. The interface of the App is now split into two sections (a) the suggested path and (b) selected artefacts which Peter can select and be directed towards them.

Peter's visit continues and finally ends without any other major reiterations of the personalisation procedure. Finally, considering Peter's previous visits which indicate an inclination to buy memorabilia from the museum's shop, the App suggests a visit at the shop, as he is exiting the museum.

\section{DISCUSSION AND FUTURE WORK}

In this research, we have extended existing work (Cheverst et al., 2000; Davies, 2007) by designing a new user personalisation method based on user personas, the CURE methodology. Its contribution focuses on the design of user personas not based on explicit input data, but on data acquisition from the user's online and social activity, thus eliminating inconsistency of user's choices or distraction of cultural experience. Furthermore, CURE methodology incorporates context aware techniques and behavioural data from the current or previous visits to cultural spaces to refine the personalization process.

Although promising, the fusion of many input streams at the personalization procedure may not deliver an accurate persona identification. Natural language processing and sentiment analysis technologies have not yet reached their maximum potential, while contextual data acquisition and reasoning may lack the precision needed in some cases. As a result, it is crucial to integrate a proof of concept component which will request user feedback and process it by adapting accordingly the persona. Overall, we are confident that the evaluation of the CURE methodology against more traditional static cultural applications will prove its merits and advantages towards a better CUX.

Also, it is worth noting that the suggested CURE framework has additional important benefits for the museum or cultural space. The satisfaction of high quality CUX offered by personalized suggestions through CURE methodology will potentially stimulate the visitor to come back and reuse the system or to encourage other people to try it as well. This can be economically advantageous for museums, which can expect an increase in virtual and real visitors as a result of CURE personalization. So, by changing the museum orientation to be more visitor-centered, based on visitors' needs, museums can be viewed as an essential cultural service.

Based on these assumptions, our future work involves the integration of CURE methodology into a cultural application. This will allow us to evaluate the effect of User Personas in Cultural User Experience and whether the suggested methodology does succeed in offering a framework for personalized applications, in the scope of cultural visits.

\section{ACKNOWLEDGMENTS}

The research and writing of this paper were financially supported by the General Secretariat for 
Research and Technology (GSRT) and the Hellenic Foundation for Research and Innovation (HFRI). John Aliprantis and Eirini Kalatha have been awarded with a scholarship for their $\mathrm{PhD}$ research from the "1st Call for PhD Scholarships by HFRI" "Grant Codes 234 \& 1347".

\section{REFERENCES}

Antoniou, A. \& Lepouras, G. (2010) Modelling visitors' profiles: A study to investigate adaptation aspects for museum learning technologies. J. Comput. Cult. Herit. 3 (2), Article No.7, pp. 1-19.

Ardissono, L., Kuflik, T. \& Petrelli, D. (2012) Personalization in cultural heritage: the road travelled and the one ahead. User Modeling and User-Adapted Interaction, 22 (1-2), pp.73-99.

Auer, S., Bizer, C., Kobilarov, G., Lehmann, J., Cyganiak, R. \& Ives, Z. (2007) DBpedia: a nucleus for a web of open data. The Semantic Web, pp. 722-735.

Bonis, B., Stamos, J., Vosinakis, S., Andreou, I. \& Panayiotopoulos, T. (2009) A platform for virtual museums with personalized content. Multimedia Tools Appl., 42 (2), pp.139-159.

Cheverst, K., Davies, N., Mitchell, K., Friday, A. \& Efstratiou, C.. (2000) Developing a Context-aware Electronic Tourist Guide: Some Issues and Experiences. Proc of $\mathrm{CHI}$ conference on Human factors in computing systems, pp.17-24.

Chianese, A. \& Piccialli, F. (2015) Improving User Experience of Cultural Environment Through loT: The Beauty or the Truth Case Study. Springer, Intelligent Interactive Multimedia Systems \& Services. Syst. Technol. 40, pp.11-20.

Davies, D.J. (2007) Improving the usability of mobile applications through context awareness. MSc Thesis, University of Oregon.

Findlater, L. \& McGrenere, J. (2004) A comparison of static, adaptive, and adaptable menus. Proceedings of the SIGCHI Conference on Human Factors in Computing Systems, CHI '04, ACM, pp.89-96.

Falk, J. H. (2006) An Identity-Centered Approach to Understanding Museum Learning. Curator: The museum journal, 49 (2), pp.151-166.

Hassenzahl, M. (2008) User Experience (UX): Towards an experiential perspective on product quality. Proceedings of the 20th Conference on I'Interaction Homme-Machine, University of Koblenz-Landau, Germany, pp.11-15,

Kenteris, M., Gavalas D. and Economou D. (2011) Electronic mobile guides: A Survey". Personal and Ubiquitous Computing, Vol.15, pp.97-111.
Konstantakis, M., Michalakis, K., Aliprantis, J., Kalatha, E. \& Caridakis, G. (2017) Formalising and Evaluating Cultural User Experience. 12th International Workshop on Semantic and Social Media Adaptation and. Personalization SMAP, IEEE Conference Publications.

Kuflik, T., Stock, O., Zancanaro, M., Gorfinkel, A., Jbara, S., Kats, S., Sheidin, J. \& Kashtan, N. (2011) A visitor's guide in an active museum: Presentations, communications, and reflection. J. Comput. Cult. H., 3 (3), Article 11.

Morris, G., Hargreaves, J. \& McIntyre, A. (2004) Learning journeys: Using technology to connect the four stages of meaning making. Birmingham: Morris, Hargreaves, McIntyre Website.

Noor, S. \& Martinez K. (2009) Using social data as context for making recommendations: an ontology based approach. In: Proceedings of the 1st Workshop on Context, Information and Ontologies, p. 7. ACM, New York.

Othman, M. K. (2012) Measuring visitors' experiences with mobile guide technology in cultural spaces. PhD Thesis, University of York.

Perera, C., Zaslavsky, A., Christen, P. \& Georgakopoulos, D. (2014) Context aware computing for the internet of things: A survey. IEEE Communications Surveys \& Tutorials, 16 (1).

Roussou, M., Katifori, A., Pujol, L., Vayanou, M. \& Rennick-Egglestone, S. J. (2013) A life of their own: museum visitor personas penetrating the design lifecycle of a mobile experience. $\mathrm{CHI}$ '13 Extended Abstracts on Human Factors in Computing Systems, ACM, New York, NY, USA, pp.547-552.

Sobhanam, H., (2013) Addressing cold start problem in recommender systems using association rules and clustering technique. International Conference on Computer Communication and Informatics (ICCCI), Coimbatore, INDIA.

Vassilakis, K., Antoniou, A., Lepouras, G., Wallace, M., Lykourentzou, I. \& Naudet, Y. (2016) Interconnecting Objects, Visitors, Sites and (Hi)Stories across Cultural and Historical Concepts: The Cross Cult project. EuroMed 2016: Digital Heritage, pp 501-510.

Vermeeren A. P., Law, E. L. C., Roto, V., Obrist, M., Hoonhout, J. \& Väänänen-Vainio-Mattila, K. (2010) User Experience Evaluation Methods: Current State and Development Needs. NordiCHI Proceedings of the 6th Nordic Conference on Human-Computer Interaction: Extending Boundaries, pp.521-530. 
Walsh, D., Clough, P. \& Foster, J. (2016) User Categories for Digital Cultural Heritage. 1st International Workshop on Accessing Cultural Heritage at Scale, USA, pp.3-9.

Wang, Y., Stash, N., Aroyo, L., Gorgels, P., Rutledge, L. \& Schreiber, G. (2008) Recommendations based on semantically enriched museum collections. Web Semantics: Science, Services and Agents on the World Wide Web, 6 (4), pp. 283-290.

Zhang, Z. K., Liu, C., Zhang, Y. C. \& Zhou, T. (2010) Solving the cold-start problem in recommender systems with social tags. EPL Journal, 92 (2). 